\title{
Research on a Frame of Cloud Service Composition in Cloud Manufacturing System
}

\author{
Min Shu ${ }^{1, a}$, Hua Guo ${ }^{2, b^{*}}$, Xiuyan Zhao ${ }^{2, c}$ \\ ${ }^{1}$ College of Mechanical and Electronic Engineering, Tanshan Polytechnic, Taian, China \\ ${ }^{2}$ College of Information Science and Engineering, Shandong Agricultural University, Taian, China \\ ashuchang198010@163.com, b*guoh@sdau.edu.cn, czhaoxy@sdau.edu.cn
}

Keywords: cloud service composition, cloud manufacturing, correlation-aware cloud service, flexibility of cloud service composition

Abstract. The cloud manufacturing (CMfg) is a new manufacturing paradigm that can realize the added-value and on-demand use of manufacturing resource and ability in the form of manufacturing cloud service (CS). In order to realize the added-value of cloud service (CS) through cloud service composition (CSC) in CMfg, there are two issues to be solved, i.e. there always exist correlations among CSs and dynamic changes during CSC, which can both affect the implement of CSC. Considering the ablve two issues, this paper presents a six-layer framework for CSC supporting life-cycle in CMfg. Meantime, the involving key issues in this framework are preliminarily discussed as well.

\section{Introduction}

The cloud manufacturing (CMfg) [1] has been widely accepted and researched both in industrial and academic communities. The existing works on CMfg primarily focus on its concept, architecture, enabling technology, etc. One of the key aims of CMfg is to realize the added-value of cloud service (CS) through cloud service composition (CSC) [2], i.e. existing CSs are assembled into a composite cloud service to serve the complex manufacturing task.

At present, there exist few researches on the CSC. However, in view of unique features of CMfg, there are two issues to be sovled. First, most existing works about QoS-based CSC treat the CSs involved in CSC as independent with each other, and their correlations are ignored. In fact, most CSs have some correlations with each other, which can affect the whole quality (i.e., QoS) of CSC badly $[3,4]$.Secondly, with the increasing of resources and CSs in CMfg CSs registry and unceasingly expansion of the scale of CSC, in view of the complexity of the process of CSC, a series of phenomenon, such as the load imbalance and network interruption, are possible to occur during CSC, as well as a variety of dynamic changes, which make the manufacturing task impossible to be completed efficiently and with high-quality. So the introduction of flexibility is an effective solution to the dynamic problem during CSC [5].

In view of these deficiencies, a framework for CSC supporting life-cycle in CMfg is designed in this paper.

\section{Related description}

Definition1 Correlation among CSs [3]. For any two CSs in the CMfg CS center, according to the influence of correlation among CSs to QoS, the correlation between two elementary CSs is defined as composable correlation, business entity correlation and statistical cooperate correlation.

Definition2 Flexibility of CSC [5]. It is defined as the ability that in the life-cycle of CSC, when CSC receives the external force (e.g. network overload) or internal force (e.g. CS exited), the resulting expected or unexpected dynamic changes can be responded and the flow of CSC can be autonomously dynamic reconfigured to complete the tasks. 


\section{The framework for cloud service composition supporting life-cycle in CMfg}

In order to implement the CSC in CMfg, this paper proposed a framework for cloud service composition supporting life-cycle in CMfg, which is shown in Fig.1. This framework is divided into six layers, i.e., resource layer, cloud service layer, task layer, CSC and optimization layer, CSC execution layer and exception process layer.

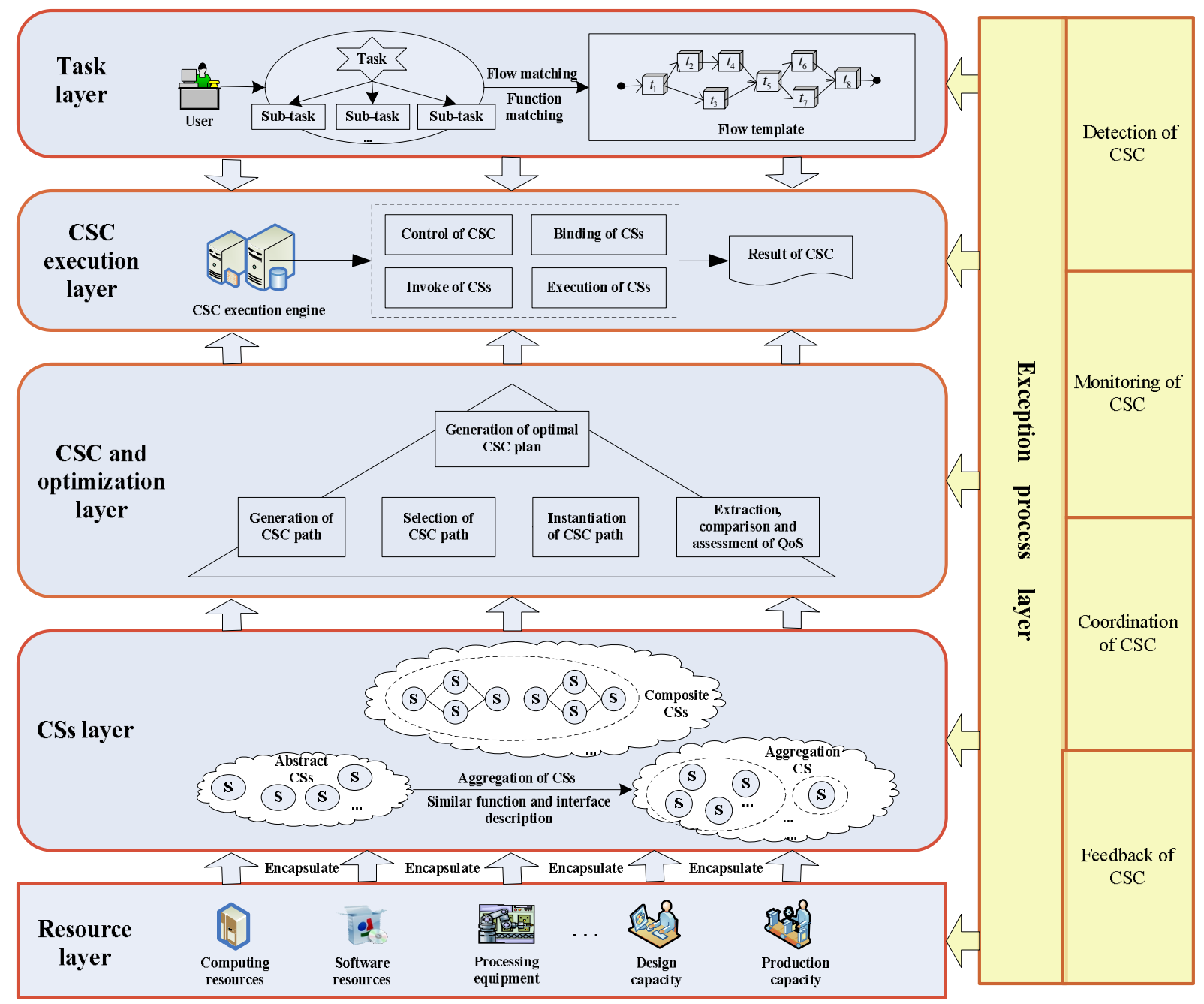

Fig.1 The framework for cloud service composition supporting life-cycle in CMfg

Resource layer. This layer provides various resources involving in the life-cycle of CSC, such as manufacturing resource and manufacturing ability, which may distribute in different place and different organization. CS providers encapsulate virtual manufacturing resources into CSs, then publishes CSs to CMfg service center to make CS requester to invoke them.

Cloud service layer. This layer mainly constructs CSs invoked by CS requester and storages CSs to CMfg services center through service-oriented encapsulation and publication of virtual resources. This layer mainly includes abstract CS, aggregation CS and composite CS. Abstract CS is encapsulated and published to the CMfg services center through various resources. Aggregation CS is those CSs that aggregated by abstract CSs that having similar functions and interface description. Composite CS is constructed by multiple abstract CSs following a certain flow. The users can select suitable CSs according to the CSs description when they invoke CSs. The layer realizes intelligent and efficient management to CSs, which can eventually provide support for the on-demand use of product lifecycle services.

Task layer. This layer mainly receives user's task request, and decomposes task into series sub-tasks. According to the flow-relationship among these sub-tasks, flow template is constructed on 
the basis of the serial, parallel, branch, cycle relationship. Then each sub-task can find corresponding abstract CSs satisfying function.

CSC and optimization layer. This layer mainly generates matching abstract CSs for each sub-task according to flow template constructed in task layer. Then candidate CS set is generated for each abstract CS through a series of operation to CSC path, such as generation of CSC path, selection of CSC path, instantiation of CSC path, and so on. Meantime, the CSs in the candidate CS set are constructed to CSC plans according to a certain logic relationship. The optimal CSC plan is selected to be executed through extraction, comparison and assessment of QoS.

CSC execution layer. This layer is to control the execution process of CSC through CSC execution engine according to selected optimal CSC plan. In addition, this layer invokes concrete CSs and complete user's task by successively executing concrete CSs. The final execution result of CSC will be submitted to user.

Exception process layer. This layer interacts with the other layers and chooses intelligent approach to carry on the process of CSC by real-time detection, monitoring, coordination, feedback and other operations, so that CSC can process the abnormal changes at any time, which can ensure normal CSC to implement.

\section{The involved key issues and solutions}

The involved key issues and solutions for cloud service composition supporting life-cycle in CMfg are analyzed as follows.

Task decomposition / demand analysis. A complex task or CS request is decomposed into a series of sub-task or activities with flow dependencies (such as serial, parallel, cycle, etc.). The function requirements and flow relationship of each sub-task are determined. The difficulty of this technology needed to overcome is how to decompose tasks automatically, and the decomposed sub-task has certain independence, as far as possible to be able to be performed by a single CS. Meantime, task decomposition granularity should be moderate, because if the particle size is too large, the degree of coupling between subtasks will increase, which may lead to the failure of follow-up function matching, flow matching and CSC. If particle size is too small, it will lead to the leap of follow-up candidate CSC plans, thereby increasing the time and space complexity of optimization of CSC plans as well as the synthesis difficulty of implementation results of CSC.

Servicization of resources. It mainly refers to related technologies involving in the process of servicization encapsulation and publication of virtual resources, which includes perception and adaptation of resources, resources virtual access, servicization of manufacturing ability, virtual resources description model based on the semantic, unified modeling, encapsulation, registering and publishing of CS, dynamic deployment and monitoring of CS, and so on.

Management of correlation among CSs. It mainly refers to related technologies in the life-cycle of CSC when considering the influence on CSC of correlation among CS, which includes description and modeling of correlation among CSs, the discovery of correlation among CSs, the assessment of correlation among CSs, updates and maintenance of correlation among CSs, and so on.

Aggregation of CSs. It refers to the operation of aggregating the CSs with similar function and interface description. If no service aggregation operation, then each CS is needed to be matched when performing service - task matching, thus reducing the efficiency of CSs to match. Through computing the similarity between CSs, CSs with similar function will be aggregated together in advance, which can improve the efficiency of follow-up CS matching.

Construction of CSC. To employ CSC to complete user's task request, the abstract CS matched by task decomposed is needed to transform to concrete CS instances, which is constructed to a executing chain structure in accordance with a certain logic flow. The involving technology is the generation of CSC paths, the selection of CSC paths, instantiation of CSC path, and so on.

Integrated processing of QoS. In the selection process of CSC, the corresponding CSs are selected according to the non-functional properties of CS, i.e. QoS. Integrated processing of QoS mainly 
addresses the following three main issues. (i) QoS information extraction, namely how to extract corresponding QoS information from the service description information. (ii) QoS evaluation. It includes QoS evaluation of a single CS as well as composite CSs, and the influence on QoS of correlation among CSs is needed to be considered. (iii) Compare of QoS and sorting of CSs based on QoS. QoS of candidate CSs and task request is mainly compared, and CSs with low quality QoS are eliminated, which can simplify difficulty and complexity of CSC and QoS optimization.

Optimal selection of CSC. There exist massive manufacturing CSs in CMfg CS platform. For each manufacturing task, a large number of candidate CSs satisfying the functional requirements may exist. Then in theory, it will form a large of CSC plans. How to select the optimal CSC plan from candidate CSC plans to execute user's task in the multi-target and multi-constraint, namely the optimal selection of CSC is key to improving quality of CSC.

CSC execution engine. It mainly refers to the technologies that can orderly process-driven CSC in accordance with logic and timing relationship, which includes the resolution of description of the document, the triggering of an operation (such as binding and invocation of a concrete service, etc.), determination of execution priority level, and so on.

Management of CSC flexibility. It mainly refers to the technologies that can process abnormal changes during CSC, which includes evaluation of CSC flexibility, detection of CSC flexibility, monitor of CSC flexibility, coordination of CSC flexibility, feedback of CSC flexibility, and so on.

\section{Conclusions}

In CMfg, there always exist correlations among CSs affecting each other, as well as the dynamic changes during CSC. Considering the correlation among CSs and CSC flexibility, this paper primarily presented a framework for CSC supporting life-cycle in CMfg, analyzed its function modules and the involving key issues. In the future, the specific solutions and algorithms for the key issues in the proposed framework will be farther investigated.

\section{Acknowledgments}

This work is partly supported by Promotive Research Fund for Excellent Young and Middle-aged Scientisits of Shandong Province (NO. BS2014ZZ012), a Project of Shandong Province Higher Educational Science and Technology Program (NO. J14LN36), and the Innovation Foundation of Shandong Agricultural University for Young Scholar.

\section{References}

[1] L.Zhang, Y.L.Luo, F.Tao, B.H.Li, L.Ren, X.S.Zhang, H.Guo, Y.Cheng, A.R.Hu, Cloud manufacturing: a new manufactring paradigam, Enterprise Information Systems, 8(2014), 167-187.

[2] F.Tao, Y.F.Hu and L.Zhang, Theory and Practice: Optimal resource service allocation in manufacturing grid, pressed by China Machine Press, China, 2010.

[3] H.Guo, F. Tao, L.Zhang, S.Y.Su and N.Si, Correlation-aware web services composition and QoS computation model in virtual enterprise, International Journal of Advanced Manufacturing Technology, 51 (2010)817-827.

[4] H.Guo, L.Zhang, Y.L.Liu, F.Tao, A discovery method of service-correlation for service composition in virtual enterprise, European Journal of Industrial Engineering, 8(2014)579-618.

[5] H.Guo, F.Tao, L.Zhang, Y.J.Laili, D.K.Liu, Research on measurement method of resource service composition flexibility in service-oriented manufacturing system, International Journal of Computer Integrated Manufacturing, 25(2012)113-135. 